\title{
Pre-asymptotic behavior of single-particle overlap integrals of non-Borromean two-neutron halos
}

\author{
N. K. Timofeyuk, ${ }^{1}$ L. D. Blokhintsev, ${ }^{2}$ and J. A. Tostevin ${ }^{1}$ \\ ${ }^{1}$ Department of Physics, School of Electronics and Physical Sciences, University of Surrey, Guildford, Surrey GU2 7XH, \\ United Kingdom \\ ${ }^{2}$ Skobeltsyn Institute of Nuclear Physics, Lomonosov State University, Moscow 117234, Russia
}

(Received 5 June 2003; published 15 August 2003)

\begin{abstract}
For non-Borromean two-neutron halo nuclei, modifications to the behavior of single-particle overlap integrals will arise due to the correlations of the two interacting nucleons in the halo. An additional contribution to the overlap integral can be obtained using the Feynman diagram approach. This additional term is modeled using a simple local potential model. We show that these modifications may play a role in detailed interpretations of experimental results from single-nucleon knockout, transfer, and other reactions that probe the single-nucleon overlap functions.
\end{abstract}

DOI: 10.1103/PhysRevC.68.021601

PACS number(s): 21.10.Jx, 24.50.+g, 27.20.+n

It is well known that when a neutron or proton is added to a stable nucleus, the separation energy of this last nucleon does not decrease monotonically. The one-nucleon separation energy $S_{A}(1 N)$ from a nucleus of mass $A$ depends on that of the previous nucleon, $S_{A-1}(1 N)$. If $S_{A}(1 N)$ $<S_{A-1}(1 N)$ then, in general, the pairing interaction between nucleons will act to increase $S_{A+1}(1 N)$. Thus, a characteristic staggering behavior is predicted theoretically and observed experimentally in the systematics of the $S(1 N)$. Near the limits of nuclear stability one finds a class of nuclei for which the one- and two-nucleon separation energies $S_{A}(1 N)$ and $S_{A}(2 N)$ are very similar, and where both are significantly smaller than those for stable nuclei. Several examples of such nuclei are shown in Table I. These weakly bound systems, with $S_{A}(2 N) \approx S_{A}(1 N)$, and for which the $A-,(A$ $-1)$-, and $(A-2)$-body nuclei are all particle stable, are referred to in this paper as non-Borromean two-neutron (or proton) halo nuclei.

Over the last decade it has been established that nuclei close to the edge of stability display shell melting and intruder states phenomena [1]. Nuclear breakup, knockout, and transfer reactions have recently been proposed [2] and used $[3,4]$ to study experimentally the strengths of the intruder states on several non-Borromean two-neutron halo systems. The theoretical interpretation of the experimental data from these reactions relies on nuclear structure and reaction theories in which the single-particle state information enters through the one-nucleon overlap integrals. An important feature of these direct reaction mechanisms is that their amplitudes are primarily sensitive to the behavior of the onenucleon overlaps at and beyond the nuclear surface. Asymptotically, these single-particle overlaps decrease exponentially with a decay constant determined by the $S_{A}(1 N)$. Most theoretical analyses of experimental data assume that these overlaps can be calculated using simple two-body potential models. These suggest that for radii outside of the range of the binding interaction, which for very weakly bound systems give the main contribution to the reaction, the asymptotic behavior for these overlaps is already achieved.

In this paper, we discuss the possibility that for nonBorromean two-nucleon halo nuclei this assumed asymptotic behavior of the one-nucleon overlaps may be reached only at much larger distances than for ordinary nuclei. The resulting changes in the overlap functions in the nuclear surface and beyond may therefore affect analyses and the interpretation of experimental results.

The physics behind this nonstandard behavior of the onenucleon overlaps is the correlations of the two nucleons of the halo outside of the nuclear core. As we attempt to remove a single nucleon to large distances $r$, beyond the range of its interactions with all the other nucleons, the remaining $A$ -1 nucleons will rearrange as if the removed nucleon was absent. In the non-Borromean two-nucleon cases of interest here, however, this $A-1$ configuration is a one-nucleon halo nucleus with a separation energy $S_{A-1}(1 N)$ considerably smaller than that of the first removed nucleon. So, for a range of $r$ beyond the nuclear core the separated nucleon will continue to overlap and interact with the halo nucleon of the $(A-1)$-body subsystem. Thus, although far from the center of mass of the $(A-1)$-body residual nucleus, the removed nucleon will be affected by these correlations of the two halo nucleons. This will lead to surface and preasymptotic deviations from the overlap functions calculated using potential models, out to large distances. These deviations may also be different for different orbital angular momenta $\ell$ of the last nucleon, and so may affect the interpretation of experiments which aim to probe the occupancies of normal and intruder single-particle states.

The one-nucleon overlap integral $I(\boldsymbol{r})$ is defined as the overlap between the (translationally invariant) many-body wave functions $\Psi_{A}$ and $\Psi_{A-1}$ of the nuclei, with masses $A$ and $A-1$, and the spin-isospin wave function $\chi_{N}$ of the removed nucleon,

$$
I(\boldsymbol{r})=\int d \tau_{A-1} \chi_{N}^{\dagger} \Psi_{A-1}^{\dagger}\left(\tau_{A-1}\right) \Psi_{A}\left(\tau_{A}\right),
$$

where $\boldsymbol{r}$ is the vector separation between the centers of mass of the $(A-1)$-body residue and the removed nucleon. Below, we consider only the $\ell=0$ case and assume that the removed nucleon is a neutron.

The best way to establish the asymptotic behavior of $I(r)$ is to consider its Fourier transform $I(q)$. The latter has a pole at imaginary momentum $q=i \kappa$, where $\kappa \equiv \kappa_{A}(1 N)$ 
TABLE I. Non-Borromean two-nucleon halo nuclei $A$, onenucleon halo nuclei $A-1$, and their common $A-2$-body cores. The two- and one-nucleon thresholds $S_{A}(2 N), S_{A}(1 N)$, and $S_{A-1}(1 N)$ are also shown.

\begin{tabular}{lccccc}
\hline \hline$A$ & $A-1$ & $A-2$ & $\begin{array}{c}S_{A}(2 N) \\
(\mathrm{MeV})\end{array}$ & $\begin{array}{c}S_{A}(1 N) \\
(\mathrm{MeV})\end{array}$ & $\begin{array}{c}S_{A-1}(1 N) \\
(\mathrm{MeV})\end{array}$ \\
\hline${ }^{12} \mathrm{Be}$ & ${ }^{11} \mathrm{Be}\left(\frac{1}{2}^{+}\right)$ & ${ }^{10} \mathrm{Be}$ & 3.670 & 3.170 & 0.500 \\
${ }^{12} \mathrm{Be}$ & ${ }^{11} \mathrm{Be}\left(\frac{1}{2}^{-}\right)$ & ${ }^{10} \mathrm{Be}$ & 3.670 & 3.490 & 0.180 \\
${ }^{15} \mathrm{~B}$ & ${ }^{14} \mathrm{~B}\left(1^{-}\right)$ & ${ }^{13} \mathrm{~B}$ & 3.740 & 3.510 & 0.230 \\
${ }^{9} \mathrm{C}$ & ${ }^{8} \mathrm{~B}(\mathrm{~g} \cdot \mathrm{s})$. & ${ }^{7} \mathrm{Be}$ & 1.433 & 1.296 & 0.137 \\
${ }^{16} \mathrm{C}$ & ${ }^{15} \mathrm{C}\left(\frac{1}{2}^{+}\right)$ & ${ }^{14} \mathrm{C}$ & 5.469 & 4.251 & 1.218 \\
${ }^{16} \mathrm{C}$ & ${ }^{15} \mathrm{C}\left(\frac{5}{2}^{+}\right)$ & ${ }^{14} \mathrm{C}$ & 5.469 & 4.991 & 0.478 \\
\hline \hline
\end{tabular}

$=\sqrt{2 \mu_{1} S_{A}(1 N)} / \hbar$ and $\mu_{1}$ is the $(A-1)+N$ reduced mass. In the absence of the Coulomb interaction, this pole provides the asymptotic behavior of $I(r)$, given by the term $[5,6]$

$$
I_{0}(r) \rightarrow C_{0} \frac{e^{-\kappa r}}{r}
$$

Graphically, this term corresponds to the point diagram of Fig. 1, panel (a). However, if a nucleus consists of more than two particles, other terms will appear in the asymptotics of $I(r)$ [6]. They arise because virtual decays into different cluster channels become possible, and are formally associated with a singular behavior of the vertex form factor $G\left(q^{2}\right) \sim\left(q^{2}+\kappa^{2}\right) I(q)$.

For non-Borromean two-neutron halo nuclei, the most important virtual decay is $A \rightarrow(A-2)+N+N$ and its contribution to the single-particle overlap integral is determined by the Feynman diagram of Fig. 1, panel (b). As has been shown in Ref. [6], for $\ell=0$ the contribution from this generalized triangle diagram is

$$
I_{1}(r)=\frac{C_{1}}{r} \int_{\kappa_{1}}^{\infty} \frac{e^{-k r}\left(k-\kappa_{1}\right)^{3 / 2}}{k^{2}-\kappa^{2}} k d k .
$$

The constant $\kappa_{1}$ is determined by the location of the singularity of diagram (b) of Fig. 1, and is equal to

$$
\kappa_{1}=\frac{(A-1)}{(A-2)}\left[\kappa_{A}(2 N)+\kappa_{A-1}(1 N)\right]
$$

Here $\kappa_{A}(2 N)$ is the momentum corresponding to the threebody virtual decay, $A \rightarrow(A-2)+N+N$,

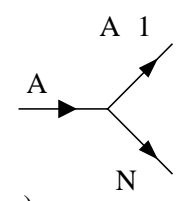

a)

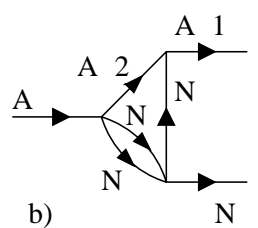

b)

$$
\kappa_{A}^{2}(2 N)=\frac{2 \mu_{2}}{\hbar^{2}} S_{A}(2 N),
$$

where $\mu_{2}$ is the $(A-2)+2 N$ reduced mass, and $\kappa_{A-1}(1 N)$ is that for the two-body virtual decay, $A-1 \rightarrow(A-2)+N$. Evaluating the integral in Eq. (3) gives

$$
\begin{aligned}
I_{1}(r)= & -\left(1-\frac{1}{2 \kappa_{1} r}\right) \frac{C_{1} \sqrt{\pi} \kappa_{1}}{r^{3 / 2}} e^{-\kappa_{1} r} \\
& +\frac{C_{1} \pi}{2 r}\left\{\left(\kappa_{1}-\kappa\right)^{3 / 2} e^{-\kappa r} \operatorname{erfc}\left[\sqrt{\left(\kappa_{1}-\kappa\right) r}\right]\right. \\
& \left.+\left(\kappa_{1}+\kappa\right)^{3 / 2} e^{\kappa r} \operatorname{erfc}\left[\sqrt{\left(\kappa_{1}+\kappa\right) r}\right]\right\},
\end{aligned}
$$

which at large distances behaves as

$$
I_{1}(r) \rightarrow \frac{C_{1}}{r} \Gamma\left(\frac{5}{2}\right) \frac{\kappa_{1}}{\kappa_{1}^{2}-\kappa^{2}} \frac{e^{-\kappa_{1} r}}{r^{5 / 2}}
$$

This behavior is reached for $r \gg\left|\kappa_{1}-\kappa\right|^{-1}$ [6].

Since for non-Borromean two-neutron halos, $S_{A-1}(1 N)$ $\ll S_{A}(2 N)$,

$$
\frac{\kappa_{1}}{\kappa} \rightarrow \sqrt{\frac{2(A-1)}{A-2}} .
$$

Because $\kappa_{1}>\kappa$, asymptotically the overlap integral $I(r)$ continues to be determined only by the term $I_{0}(r)$. In practice, in the analysis of experiments, calculations are sensitive to smaller distances where the contribution from $I_{1}(r)$ may be non-negligible. For the typical three-body energies of the non-Borromean two-nucleon halo systems shown in Table I, $\kappa$ lies in the range of $0.25-0.5 \mathrm{fm}^{-1}$. In the most extreme case of $S_{A-1}(1 N) \ll S_{A}(2 N), \kappa_{1}$ can be larger than $\kappa$ by only $40 \%$ and the ratio $I_{1}(r) / I_{0}(r)$ decreases only slowly in the region between 5 and $10 \mathrm{fm}$ (see Fig. 2) of importance to reaction analyses. Clearly, the surface contributions from $I_{1}(r)$ depend critically on the strength of the coupling constant $C_{1}$.
FIG. 1. The point (a) and generalized triangle (b) Feynman diagrams for the $A \rightarrow(A-1)+N$ vertex. 
To calculate $C_{1}$ from the many-body overlap, Eq. (1), the wave function $\Psi_{A}$ must have the correct asymptotic in the three-body channel to provide the correct analytical properties for diagram (b) of Fig. 1. Available many-body approaches cannot generate these correct three-body asymptotics, although, existing many-body models could be used to calculate $C_{1}$ as the discontinuity of the amplitude of diagram (b). To do this, the Feynman integral of the product of four propagators and three vertex functions should be written and then modified to include not only the $A^{-},(A-1)$-, and ( $A$ -2)-body nuclear wave functions, but pairwise potentials too, in order to cut off unreliable long-range contributions. Developments along these lines will constitute a major longterm project.

In the absence of a proper many-body approach, in this paper we study possible pre-asymptotic changes to the overlap integrals for non-Borromean two-neutron halos within a simple two-body potential model. We use an effective local potential $V_{e f f}(r)$ which, for a given $C_{1} / C_{0}$, generates an asymptotic behavior for the overlap $I(r)$ given by the sum of Eqs. (2) and (6). The long-range nature of such a potential can be obtained from the Schrödinger equation

$$
\left(\frac{d^{2}}{d r^{2}}-\kappa^{2}\right)\left[r I_{a s}(r)\right]=\frac{2 \mu_{1}}{\hbar^{2}} V_{e f f}(r)\left[r I_{a s}(r)\right]
$$

with $I_{a s}(r)=I_{0}(r)+I_{1}(r)$. It can be shown that

$$
V_{e f f}(r)=-\frac{\hbar^{2}}{2 \mu_{1}} c_{10} \sqrt{\frac{\pi}{\kappa_{1}}} \frac{e^{-\left(\kappa_{1}-\kappa\right) r}}{r^{5 / 2}} \frac{1+\frac{5}{2 \kappa_{1} r}}{1+\frac{I_{1}(r)}{I_{0}(r)}} .
$$

Here $c_{10}=\kappa_{1}^{3 / 2} C_{1} / C_{0}$ is a dimensionless constant. One can see that at large $r$, where $I_{0}$ dominates, this potential decreases as $e^{-\left(\kappa_{1}-\kappa\right) r} / r^{5 / 2}$, the rate of decrease determined by $\kappa_{1}-\kappa$. In the extreme case of Eq. (8), $\kappa_{1}-\kappa \approx 0.4 \kappa$ and can range between 0.1 and $0.2 \mathrm{fm}^{-1}$, providing a very slow decrease. For small values of $c_{10}$, the effective potential, Eq. (10), is proportional to $c_{10}$, while for very large $c_{10}$, when $I_{1}(r) / I_{0}(r) \gg 1, V_{\text {eff }}(r)$ is independent of $c_{10}$ and is determined only by $\kappa$ and $\kappa_{1}$. This places an upper limit for $V_{\text {eff }}(r)$.

We have calculated the potentials $V_{\text {eff }}(r)$ in the extreme case of Eq. (8) for two values of $\kappa, 0.25$ and $0.5 \mathrm{fm}^{-1}$, and for a range of $c_{10}$, shown in Fig. 3. For comparison, standard Woods-Saxon potentials, with the depth of $50 \mathrm{MeV}$, radius of $1.25 A^{1 / 3} \mathrm{fm}$, and diffuseness of $0.65 \mathrm{fm}$, are also shown in Fig. 3 for $10 \leqslant A \leqslant 100$, and which decrease much faster. For each $A$, a minimum value of $c_{10}$ exists for which the longrange effective potentials $V_{\text {eff }}(r)$ compete with the WoodsSaxon potential in the near-surface region. These $c_{10}$ values increase with $A$. One should therefore expect that the largest possible effects of any preasymptotic deviations will be manifested in the lightest nuclei, for example, in the $\left\langle{ }^{11} \mathrm{Be}(\mathrm{g} . \mathrm{s}) \mid.{ }^{12} \mathrm{Be}\right\rangle$ overlap. For heavy nuclei these effects might only be noticeable for $c_{10} \gg 1$.
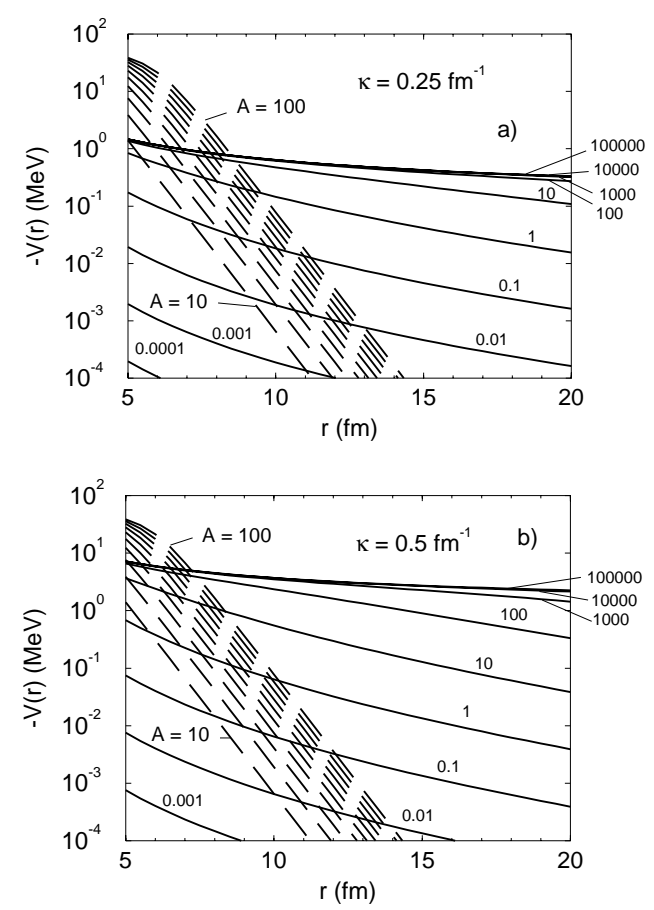

FIG. 3. Effective local potentials $V_{e f f}(r)$ (solid lines) for different values of $c_{10}$, shown by numbers next to solid lines, for (a) $\kappa$ $=0.25 \mathrm{fm}^{-1}$ and (b) $\kappa=0.5 \mathrm{fm}^{-1}$, compared to standard WoodsSaxon potentials (dashed lines), for $10 \leqslant A \leqslant 100$.

Figure 3 suggests that, to model the preasymptotic behavior within a two-body potential model, one can introduce a model potential $V_{\text {model }}(r)$ consisting of two parts, $V_{\text {core }}(r)$ and $V_{\text {halo }}(r) . V_{\text {halo }}(r)$ here accounts for the interaction of the removed neutron with the halo neutron of the residual nucleus, $A-1$. We give it a radial form similar to the Woods-Saxon potential,

$$
V_{\text {halo }}(r)=-V_{0 h}\left(1+\frac{\alpha r^{5 / 2}}{1+\frac{5}{2 \kappa_{1} r}} e^{\left(r-R_{h}\right) / a_{h}}\right)^{-1},
$$

which is approximately constant for small $r$ and which approximates $V_{\text {eff }}(r)$ outside the nucleus. $V_{\text {halo }}(r)$ has the asymptotic behavior given by Eq. (10). Potential $V_{\text {core }}(r)$ models the interaction of the removed nucleon and the $(A$ -2)-body core of the residual mass $A-1$ nucleus. It is of short range and is chosen to be of Woods-Saxon form, $V_{\text {core }}(r)=-V_{0 c} /\left(1+\exp \left(\left[r-R_{c}\right] / a_{c}\right)\right)$, with standard radius and diffuseness parameters, see Table II.

As an example, we have taken the $\left\langle{ }^{11} \mathrm{Be}\right.$ (g.s.) $\left.\mid{ }^{12} \mathrm{Be}\right\rangle$ overlap, which enters the analysis of the one-nucleon knockout reaction, such as has been measured in Ref. [3]. For this overlap, $\kappa=0.372 \mathrm{fm}^{-1}$ and $\kappa_{1}=0.766 \mathrm{fm}^{-1}$. We have calculated the tails of the effective potentials $V_{\text {eff }}(r)$ for different $c_{10}$ using Eq. (10). In Fig. 4, these are compared with the conventional Woods-Saxon potential which binds the neutron in ${ }^{12} \mathrm{Be}$ with the correct separation energy. We note that for $c_{10}<1$ the long-range contribution from $V_{\text {eff }}(r)$ is too small to compete with the Woods-Saxon potential or to pro- 
TABLE II. The ratios $c_{10}$ and the parameters $V_{0 h}, \alpha, R_{h}, a_{h}$ of the potential $V_{\text {halo }}$. Also shown are the depths $V_{0 c}$ of the potential $V_{\text {core }}$ and the asymptotic normalization coefficients $b=r \varphi_{\text {model }}(r) / e^{-\kappa r}, r \rightarrow \infty$.

\begin{tabular}{lcccccc}
\hline \hline$c_{10}$ & $\begin{array}{c}V_{0 h} \\
(\mathrm{MeV})\end{array}$ & $\begin{array}{c}\alpha \\
\left(\mathrm{fm}^{-5 / 2}\right)\end{array}$ & $\begin{array}{c}R_{h} \\
(\mathrm{fm})\end{array}$ & $\begin{array}{c}a_{h} \\
(\mathrm{fm})\end{array}$ & $\begin{array}{c}V_{0 c} \\
(\mathrm{MeV})\end{array}$ & $\begin{array}{c}b \\
\left(\mathrm{fm}^{-1 / 2}\right)\end{array}$ \\
\hline 0 & 0 & & & & 65.505 & 2.610 \\
1 & 14.86 & 3.275 & 8.214 & 3.881 & 55.477 & 2.638 \\
2 & 14.84 & 2.067 & 9.121 & 3.899 & 52.848 & 2.271 \\
10 & 14.70 & 3.112 & 17.26 & 4.037 & 43.315 & 3.269 \\
100 & 13.81 & 1.123 & 25.42 & 5.050 & 21.538 & 7.523 \\
\hline \hline
\end{tabular}

duce significant deviations to the $\left\langle{ }^{11} \mathrm{Be}(\mathrm{g} . \mathrm{s}) \mid.{ }^{12} \mathrm{Be}\right\rangle$ overlap. For each $c_{10}>1$ we have fitted the calculated $V_{\text {eff }}(r)$ for $r$ $>5 \mathrm{fm}$ to the form of the potential $V_{\text {halo }}$ of Eq. (11). The deduced values of $V_{0 h}, \alpha, R_{h}$, and $a_{h}$ are collected in Table II. For each of these potentials we have fitted the depth $V_{0 c}$ of $V_{\text {core }}$ to reproduce the physical neutron separation energy, assuming that $R_{c}=2.78 \mathrm{fm}$ and $a_{c}=0.65 \mathrm{fm}$. The $V_{0 c}$ are also shown in Table II.

The single-particle wave functions $\varphi_{\text {model }}(r)$ generated by the $V_{\text {model }}$ potentials from Table II and their ratios to the true asymptotic form $e^{-\kappa r} / r$ are shown in parts (a) and (b), respectively of Fig. 5. The deviations from the usual asymptotic behavior are significant for the values of $c_{10}>2$ and increase with $c_{10}$.

We have calculated the cross sections for the one-neutron removal reaction from ${ }^{12} \mathrm{Be}$, using the eikonal reaction theory methods of Refs. [3,7], and using the model overlaps calculated with $c_{10}$ of $0,2,10$, and 100 . The diffractive breakup, stripping, and their summed cross sections and the longitudinal momentum distributions of the ${ }^{11} \mathrm{Be}$ (g.s.) residues are shown in parts (a) and (b), respectively, of Fig. 6. The neutron removal cross sections calculated with the wave functions $\varphi_{\text {model }}(r)$ corresponding to $c_{10}$ of 2,10 , and 100 are larger than those calculated with $c_{10}=0$ by $3 \%, 22 \%$, and $124 \%$, respectively. As for the longitudinal distributions, they become narrower with the increase of $c_{10}$. For $c_{10}<10$, their shapes agree with the experimental ones, taken from Ref. [3], within the present error bars. So, within the context of the eikonal reaction theory used [3,7], the constraint $c_{10}<10$ is

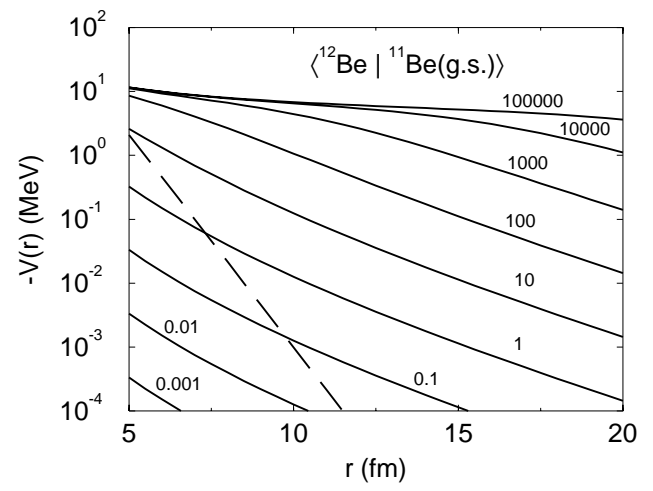

FIG. 4. Effective local potentials $V_{\text {eff }}(r)$ (solid lines) for different $c_{10}$ values, for the $\left\langle{ }^{11} \mathrm{Be}(\mathrm{g} . \mathrm{s}) \mid.{ }^{12} \mathrm{Be}\right\rangle$ overlap, compared with a standard Woods-Saxon potential model (dashed line). consistent with the experimental data. On the basis of Fig. 6(a), this, in turn, would imply an upper bound on the uncertainty in spectroscopic factors due to such preasymptotic abnormalities of the order of $22 \%$.

For the $\left\langle{ }^{11} \mathrm{Be}\left(\frac{1}{2}^{-}\right) \mid{ }^{12} \mathrm{Be}\right\rangle$ overlap, which is relevant to the $\ell=1$ neutron knockout leading to the ${ }^{11} \mathrm{Be}$ residue in the $\frac{1}{2}^{-}$ first excited state, the value of $\kappa_{1}$, equal to $0.577 \mathrm{fm}^{-1}$, is smaller than that in the case of the ${ }^{11} \mathrm{Be}$ (g.s.) transition. It follows that, for fixed $c_{10}$, the effective potentials $V_{\text {eff }}(r)$, if still assumed given by Eq. (13), are larger. These increased deviations are compensated somewhat by the centrifugal potential, but are still larger than those in the $\ell=0$ case. For $c_{10}=10$, the change to the shape of the longitudinal momentum distribution is essentially the same as for the $\ell=0$ case, however the calculated cross section now increases by $41 \%$. More definite conclusions will be possible only after proper calculation of the $V_{e f f}(r)$ for the $\ell=1$ case.
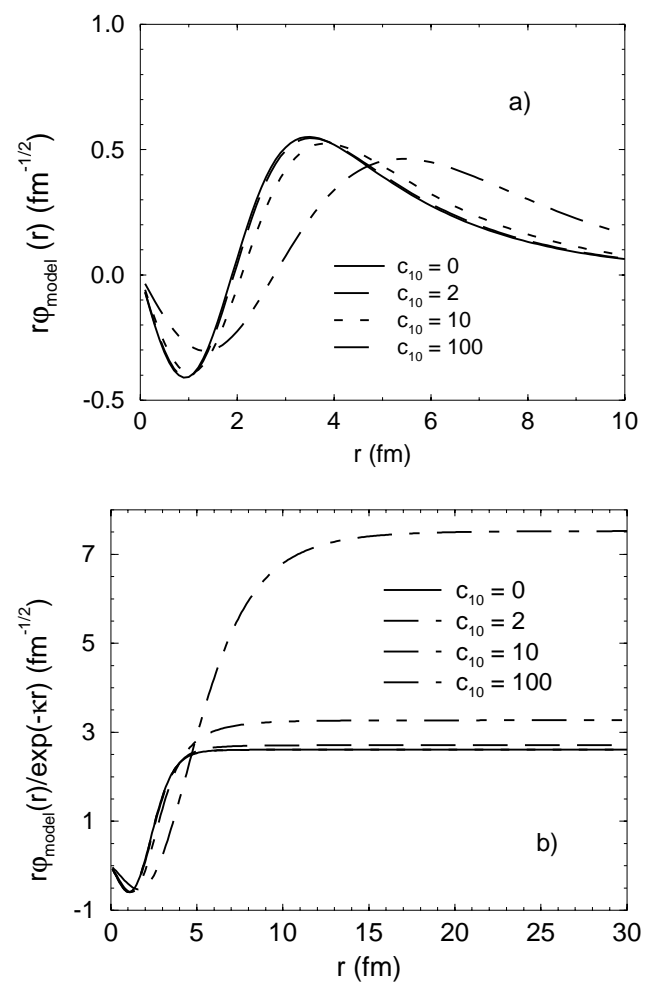

FIG. 5. (a) The single-particle wave functions $r \varphi_{\text {model }}(r)$ generated by the potentials $V_{\text {model }}(r)$, and (b) their ratios to the true asymptotics $e^{-\kappa r}$, calculated for different values of $c_{10}$. 

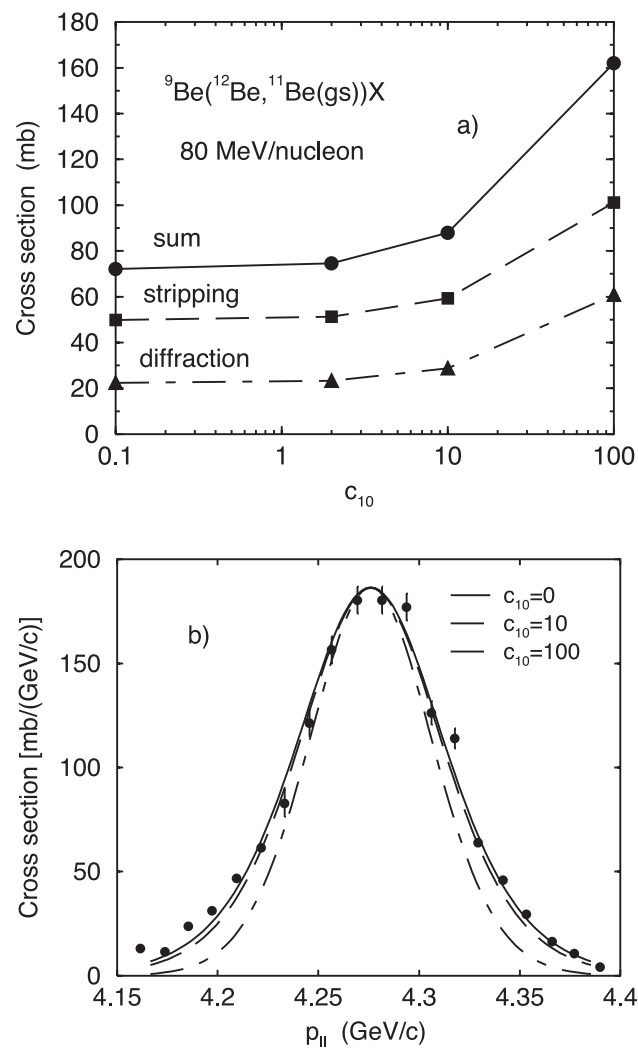

FIG. 6. (a) Partial cross section contributions, and (b) longitudinal momentum distributions from one-neutron knockout from

${ }^{12} \mathrm{Be}$ populating ${ }^{11} \mathrm{Be}$ (g.s.) residues, calculated for different values of $c_{10}$. The data are taken from Ref. [3].

The example of ${ }^{12} \mathrm{Be}$ shows that possible irregularities in the preasymptotic behavior of the single-particle overlap integrals for non-Borromean two-neutron halo nuclei deserve serious theoretical study. This requires development of many-body nuclear structure models with an explicit asymptotic treatment of three-body channels, together with the Feynman integral techniques. A careful analysis at the level of a three-body model, with binary channels, would also be very helpful.

The presence of significant irregularities in the singleparticle overlaps for non-Borromean halos, if confirmed, would have important consequences for direct $(p, \gamma)$ and $(n, \gamma)$ capture reactions in which non-Borromean twonucleon halos are produced on loosely bound even-odd nuclei in the $r p$ and $r$ processes. The amplitudes of these reactions include the same overlap integrals that appear in onenucleon removal reactions studied in terrestrial laboratories. At astrophysical energies, the $(p, \gamma)$ reactions occur at the region of a true asymptotic behavior of overlap integrals given by a point diagram (a) and their cross sections are determined by normal asymptotic normalizing coefficients (ANCs). The $(n, \gamma)$ reactions can be sensitive for any part of the overlap integral depending on the target nucleus. At present, simple two-body potential models, in which extranuclear behavior of the wave functions is normal, are used to calculate the low-energy cross sections of such reactions. The results obtained within these models are often normalized according either to ANCs or to spectroscopic factors determined experimentally from transfer, breakup, or knockout reactions. Although such reactions are often peripheral, they are sensitive to the extranuclear but near-surface part of the single-particle overlap integral that can be influenced by preasymptotic abnormalities. If the latter are significant, then what is extracted from such reactions may not correspond to the true ANCs or spectroscopic factors. A particular example is the ${ }^{8} \mathrm{~B}(p, \gamma){ }^{9} \mathrm{C}$ reaction. It has been studied recently indirectly via knockout $[8,9]$ and transfer reactions [10] and in a simple potential model [11]. The situation is aggravated here by the long-range Coulomb interaction between the last two protons, which may lead to stronger pre-asymptotic abnormalities. Further study of the pre-asymptotic behavior of single-particle overlaps for non-Borromean two-neutron and two-proton halos is an urgent and timely task.

This work was supported by the United Kingdom Engineering and Physical Sciences Research Council (EPSRC), Grant No. GR/M82141.
[1] B.A. Brown, Prog. Part. Nucl. Phys. 47, 517 (2001).

[2] F.M. Nunes, J.A. Christley, I.J. Thompson, R.C. Johnson, and V.D. Efros, Nucl. Phys. A609, 43 (1996).

[3] A. Navin et al., Phys. Rev. Lett. 85, 266 (2000).

[4] V. Maddalena et al., Phys. Rev. C 63, 024613 (2001).

[5] L.D. Blokhintsev, I. Borbely, and E.I. Dolinskii, Sov. J. Part. Nucl. 8, 485 (1977).
[6] L.D. Blokhintsev, Bull. Russ. Acad. Sci. Phys. 65, 77 (2001).

[7] J.A. Tostevin, Nucl. Phys. A682, 320c (2001).

[8] L. Trache, F. Carstoiu, A.M. Mukhamedzhanov, and R.E. Tribble, Phys. Rev. C 66, 035801 (2002).

[9] J. Enders et al., Phys. Rev. C 67, 064301 (2003).

[10] D. Beaumel et al., Phys. Lett. B 514, 226 (2001).

[11] P. Mohr, Phys. Rev. C 67, 065802 (2003). 